\title{
Mechanics of finger-tip electronics
}

\author{
Yewang Su, ${ }^{1,2, a)}$ Rui Li, ${ }^{3, a)}$ Huanyu Cheng, ${ }^{2}$ Ming Ying, ${ }^{4}$ Andrew P. Bonifas, ${ }^{4}$ \\ Keh-Chih Hwang, ${ }^{1}$ John A. Rogers, ${ }^{4, b)}$ and Yonggang Huang ${ }^{2, b)}$ \\ ${ }^{1}$ Center for Mechanics and Materials, Tsinghua University, Beijing 100084, China \\ ${ }^{2}$ Department of Civil and Environmental Engineering, Department of Mechanical Engineering, \\ Center for Engineering and Health and Skin Disease Research Center, Northwestern University, \\ Evanston, IL 60208 \\ ${ }^{3}$ State Key Laboratory of Structural Analysis for Industrial Equipment, Department of Engineering Mechanics, \\ Dalian University of Technology, Dalian 116024, China \\ ${ }^{4}$ Department of Materials Science and Engineering, Frederick Seitz Materials Research Laboratory, \\ University of Illinois at Urbana-Champaign, Urbana, IL 61801, USA
}

(Received 9 September 2013; accepted 14 October 2013; published online 31 October 2013)

\begin{abstract}
Tactile sensors and electrotactile stimulators can provide important links between humans and virtual environments, through the sensation of touch. Soft materials, such as low modulus silicones, are attractive as platforms and support matrices for arrays sensors and actuators that laminate directly onto the fingertips. Analytic models for the mechanics of three dimensional, form-fitting finger cuffs based on such designs are presented here, along with quantitative validation using the finite element method. The results indicate that the maximum strains in the silicone and the embedded devices are inversely proportional to the square root of radius of curvature of the cuff. These and other findings can be useful in formulating designs for these and related classes of body-worn, three dimensional devices. (C) 2013 AIP Publishing LLC.
\end{abstract}

[http://dx.doi.org/10.1063/1.4828476]

\section{INTRODUCTION}

Tactile sensors and electrotactile stimulators mounted on the fingertips can establish natural connections between a human subject and a virtual environment. Such devices, when designed using ultrathin, skin-like properties, offer the potential to significantly improve interfaces needed in simulated surgery, therapeutic devices, and robotic manipulators. ${ }^{1-3}$ Tactile sensors measure the pressure created by physical contact. Together with motion detection and temperature sensing, such can serve in feedback loops with electrotactile stimulators that create artificial sensations of touch, commonly perceived as a vibration or tingling feeling. ${ }^{4,5}$ Related technologies have been explored for programmable braille readers and displays for the visually impaired as well as for balance control in individuals who suffer from vestibular disorders. ${ }^{3,6-9}$

The concepts of stretchable electronics have potential utility in these contexts. ${ }^{10-14}$ In particular, they create opportunities to incorporate tactile sensors and electrotactile stimulators into conformal, skin-like platforms that intimately, and non-invasively mount on the fingertips. Ying et al. ${ }^{15}$ reported materials, fabrication strategies and device designs for ultrathin, stretchable silicon-based electronics and sensors that can be mounted on the inner and outer surfaces of soft, and elastomeric closed-tube structures for integration directly on the fingertips. The active components and interconnects incorporate advanced mechanics designs, capable of accommodating large strains induced not only by natural deformations of the soft tubes during use but also during a

\footnotetext{
${ }^{\text {a) }}$ Y. Su and R. Li contributed equally to this work.

b) Authors to whom correspondence should be addressed. Electronic addresses: y-huang@northwestern.edu and jrogers@illinois.edu.
}

critical step in the fabrication process in which the soft tubes, specially formed to match the shapes of fingertips, were flipped inside-out, as illustrated in Fig. 1. Figure 1(a) shows a finger-tube made of a very soft silicone elastomer (Ecoflex, Smooth-On, Inc.) in three dimensional forms specifically matched to those of fingers on a plastic model of the hand. The fabrication involves pouring a polymer precursor to Ecoflex onto a model finger multiple times, to create a conformal sheet with $\sim 500 \mu \mathrm{m}$ thickness. As illustrated in Fig. 1(b), removing the Ecoflex from the model forms a free standing finger-tube shown in Fig. 1(c). Ecoflex is an attractive material for this purpose because it has a low modulus $(\sim 60 \mathrm{kPa})$ and large fracture strain $(\sim 900 \%)$. The former allows soft, intimate contact with the skin; the latter enables the above "flipping-over" process. Transfer printing delivers the electrotactile stimulators to the outer surface of the flipped finger-tube, while pressed into a flattened geometry (Fig. 1(c)). The flipped finger-tube (with the electrotactile stimulators on the outer surface) is then slipped onto the finger model (Fig. 1(d)), and is flipped inside-out, to move the electrotactile stimulators from the outer to the inner surface of the tube, as shown in Fig. 1(e).

This "flipping-over" process allows devices initially mounted on the outer surface of the soft tube to be reversed to the inner surface, where they can press directly against the skin when mounted on the fingers. These concepts in multifunctional fingertip devices were demonstrated with electrotactile electrode arrays multiplexed with Si nanomembrane (NM) diodes, strain sensors based on Si NM gauges, and tactile sensor arrays that use capacitors with low-modulus, elastomeric dielectrics.

This paper aims at establishing analytic mechanics models that determine the maximum strain in the "flipping-over" 

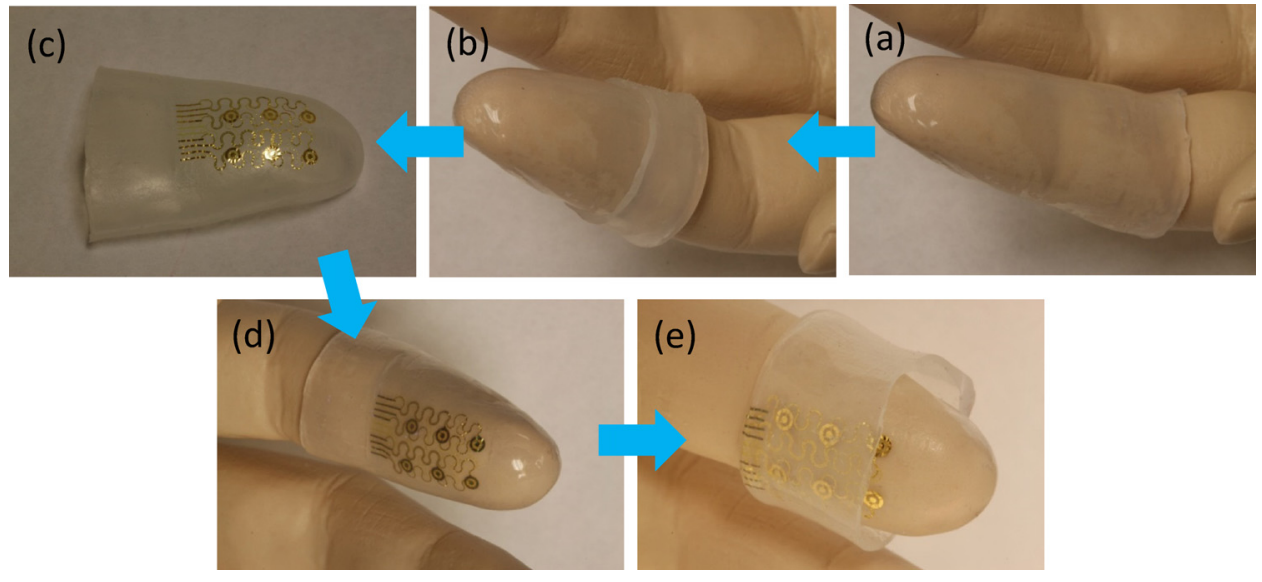

FIG. 1. The "flipping over" process in the fabrication of finger-tip electronics: (a) a finger-tube at the natural, stressfree state on a plastic model of the hand; (b) a "flipping-over" process releases the finger-tube from the model to expose its inner surface; (c) the flipped finger-tube with the electrotactile stimulators on the outer surface; (d) the finger-tube slipped onto the finger model; (e) the "flipping-over" process for the second time moves the electrotactile stimulators from the outer to the inner surface. process. The finite element method (FEM) is used to validate the results. The predicted shape after the "flipping-over" process agrees well with experiments. A scaling law for the maximum strain in the device is established, which is useful in optimizing the design of devices to survive the "flippingover" process used to fabricate fingertip electronics.

\section{AN ANALYTIC MODEL FOR SOFT MATERIALS IN FINGERTIP ELECTRONICS}

The schematic diagrams in Fig. 2 further illustrate the "flipping-over" process by tracking points $a$ - $h$ on the inner and outer surfaces of the finger-tube, where Figures 2(a)-2(e) correspond to Figures 1(a)-1(e), respectively. Figure 2(a) shows a soft, ecoflex finger-tube at the natural, stress-free state, with points $a$ - $d$ on the inner and $e-h$ on the outer surfaces, respectively, of the finger-tube. The soft finger-tube is flipped over (Fig. 2(b)) to expose the inner surface out and form a free standing finger-tube shown in Fig. 2(c), where points $a-d$ and $e-h$ switch to be on the outer and inner surfaces of the flipped finger-tube, respectively. As to be shown in the following, the electrotactile stimulators transfer printed to the outer surface of the flipped finger-tube (while pressed into a flattened geometry) have very low stiffness. They have little effect on the "flipping-over" process, and are neglected in the analytic model. The flipped finger-tube (with the electrotactile stimulators on the outer surface) is then slipped onto the finger model (Fig. 2(d)), and points $a-d$ and $e-h$ remain on the outer and inner surfaces, respectively. The soft finger-tube is then flipped over again (Fig. 2(e)) such that the electrotactile stimulators are on the inner surface and the strain gauge arrays and tactile sensors are transfer printed on the outer surface (while pressed into a flattened geometry).

Figure 3 shows an axisymmetric model for the flipped finger-tube, where $R_{\text {finger }}$ is the finger radius, $t$ is the fingertube thickness, $a$ and $d$ represent the last section of contact, and $(r, z)$ are the axisymmetric coordinates. The contact length $L$ with the finger, the bend radius $R$ of the finger-tube, and contact length $L^{\prime}$ of the "flipped over" part (Fig. 3) are to be determined. The flipped figure tube consists of four parts, with the corresponding profile $r=r(z)$ for their mid planes given in the following:

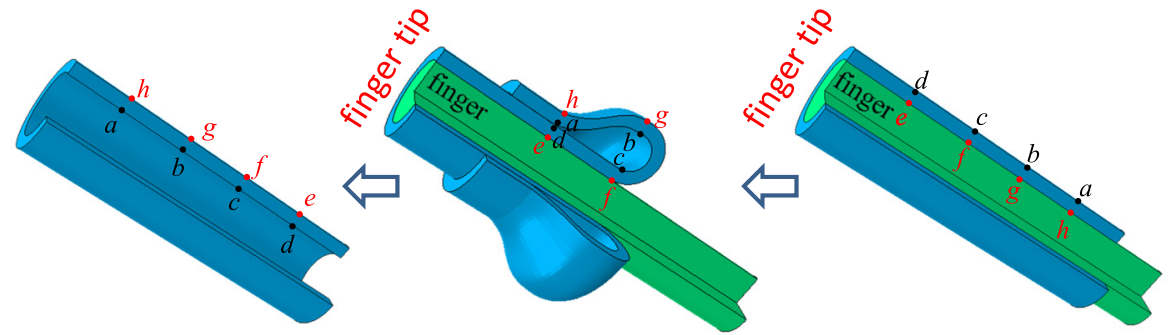

(c) after first flipping over

(b) first flipping over

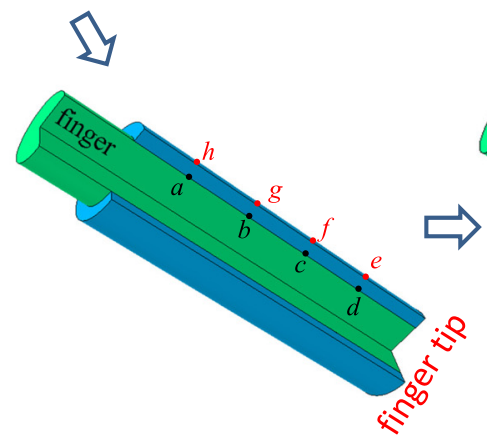

(d) slipping onto the finger (a) stress free

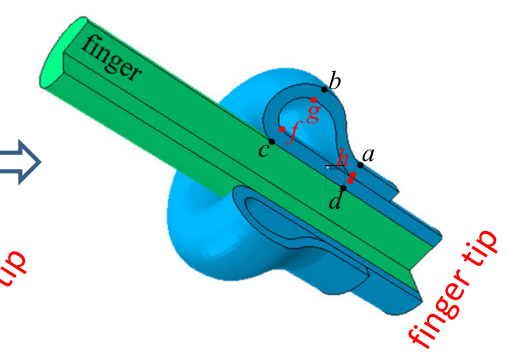

(e) second flipping over
FIG. 2. The schematic diagrams of the "flipping-over" process by tracking points $\mathrm{a}-\mathrm{h}$ on the inner and outer surfaces of the finger-tube, where Figures 2(a)-2(e) correspond to Figures 1(a)-1(e), respectively. 


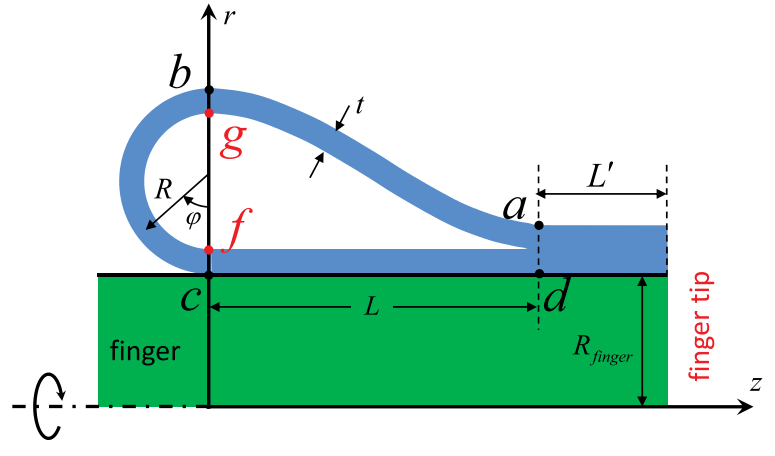

FIG. 3. Schematic illustration of the axisymmetric model for the flipped finger-tube.

(1) Part $c d$ (and beyond), which is a straight line in Fig. 3 (and a cylinder wrapping around the finger in 3D), with $r=R_{\text {finger }}+(t / 2)$ for $0 \leq z \leq L+L^{\prime}$.

(2) Part $b c$, which is approximated by a semicircle of radius $R$ (to be determined) in Fig. 3, with $\left[r-R_{\text {finger }}-R\right.$ $-(t / 2)]^{2}+z^{2}=R^{2}$ for $-R \leq z \leq 0$, or equivalently $r=R_{\text {finger }}+R+(t / 2) \pm \sqrt{R^{2}-z^{2}}$, where \pm are for the upper and lower halves, respectively.

(3) Part $a b$, which is approximated by a sinusoidal curve with continuous slopes at its ends, $r=R_{\text {figure }}+R+t$ $+(R-t / 2) \cos (\pi z / L)$ for $0 \leq z \leq L \quad(L \quad$ is $\quad$ to be determined).

(4) The "straight" part beyond $a$, which is a straight line in Fig. 3 (and a cylinder in 3D), with $r=R_{\text {finger }}+(3 t / 2)$ for $L \leq z \leq L+L^{\prime}$.

Here the axisymmetric coordinates $(r, z)$, rather than the Gaussian coordinates in differential geometry for a curvilinear surface are used for convenience. The total length of the finger-tube is given by

$$
L_{\text {total }}=L+\pi R+\int_{0}^{L} \sqrt{1+\frac{\pi^{2}}{L^{2}}\left(R-\frac{t}{2}\right)^{2} \sin ^{2}\left(\frac{\pi z}{L}\right)} d z+2 L^{\prime}
$$

where the integral represents the arc length of part $a b$, which can be obtained analytically as $(2 R-t) E(\zeta) / \zeta$, where $E(\zeta)$ is the complete elliptic integral of second kind, ${ }^{16}$ and $\zeta=\left\{1+\left(L^{2} / \pi^{2}\right)[R-(t / 2)]^{-2}\right\}^{-1 / 2}$. Equation (1) gives $L^{\prime}$ in terms of $L$ and $R$ for a given total length $L_{\text {total }}$.

\section{A. Curvatures and bending energy}

The principal curvature along the meridional direction is $\kappa_{s}=\left(d^{2} r / d z^{2}\right) /\left[1+(d r / d z)^{2}\right]^{3 / 2}$ at any point $[r(z), z]$ on the surface. The other principal curvature $\kappa_{\theta}$ along the circumferential direction is the negative reciprocal of the distance from the point $[r(z), z]$ along its normal to the point of intersection with the axis of symmetry $z=0$, and is given by $-\left[r{\sqrt{1+(d r / d z)^{2}}}^{-1}\right.$. The corresponding initial curvatures in Fig. 2(a) are $\kappa_{s 0}=0$ and $\kappa_{\theta 0}= \pm\left[R_{\text {finger }}+(t / 2)\right]^{-1}$, where + is for part $c d$ and the lower half of part $b c$, and - is for the upper half of part $b c$, and part $a b$ and beyond. Here the opposite signs are because points $a$ to $b$ and $c$ to $d$ have the same directions (toward the finger tip) in Fig. 2(a), and opposite directions in Fig. 2(e) (and Fig. 3) due to "flipping over" of the part $a b$ and upper half of part $b c$. The above profile $r=r(z)$ gives the principal curvatures as

(1) Part $c d$ (and beyond): $\kappa_{s}=0$ and $\kappa_{\theta}=-\left[R_{\text {finger }}\right.$ $+(t / 2)]^{-1}$.

(2) Part $b c: \kappa_{s}= \pm R^{-1}$, where + and - are for the lower and upper halves of part $b c$, respectively, and $\kappa_{\theta}=-\sqrt{R^{2}-z^{2}} /(R r)$.

(3) Part $\quad a b$ : $\quad \kappa_{s}=-\left(\pi^{2} / L^{2}\right)[R-(t / 2)] \cos (\pi z / L) /\{1$ $\left.+\left(\pi^{2} / L^{2}\right)[R-(t / 2)]^{2} \sin ^{2}(\pi z / L)\right\}^{3 / 2}$ and $\kappa_{\theta}=-\left\{R_{\text {figure }}\right.$ $+R+t+[R-(t / 2)] \cos (\pi z / L)\}^{-1}\left\{1+\left(\pi^{2} / L^{2}\right)[R-(t / 2)]^{2}\right.$ $\left.\sin ^{2}(\pi z / L)\right\}^{-1 / 2}$.

(4) The "straight" part beyond $a: \kappa_{s}=0$ and $\kappa_{\theta}=-\left[R_{\text {finger }}\right.$ $+(3 t / 2)]^{-1}$.

Here the curvature in Part $b c$ has opposite signs for the lower and the upper halves because of the use of axisymmetric coordinates $(r, z)$. The Gaussian coordinates would give the same sign for the curvature, but they are inconvenient to describe all four parts of the flipped finger-tube.

The finger-tube is modeled as a thin shell with the Young's modulus $E$ and thickness $t$. The bending energy density is ${ }^{17}$

$$
\begin{aligned}
u_{b}= & \frac{D}{2}\left[\left(\kappa_{s}-\kappa_{s 0}\right)^{2}+\left(\kappa_{\theta}-\kappa_{\theta 0}\right)^{2}\right. \\
& \left.+2 \nu\left(\kappa_{s}-\kappa_{s 0}\right)\left(\kappa_{\theta}-\kappa_{\theta 0}\right)\right],
\end{aligned}
$$

where $D=E t^{3} /\left[12\left(1-\nu^{2}\right)\right]$ is the bending stiffness and $\nu$ is the Poisson's ratio. The bending energy in the shell is the integration of $u_{b}$ over the area of finger-tube, and is given by

$$
\begin{aligned}
& U_{b}=2 \pi\left(R_{\text {finger }}+\frac{t}{2}\right) *\left[\int_{0}^{L+L^{\prime}} u_{b}^{(c d)} d z\right. \\
& +\int_{-R}^{0}\left[u_{b}^{(b c)_{\text {lower }}}+u_{b}^{(b c)_{\text {upper }}}\right] \sqrt{1+\left(\frac{d r}{d z}\right)^{2}} d z \\
& \left.+\int_{0}^{L} u_{b}^{(a b)} \sqrt{1+\left(\frac{d r}{d z}\right)^{2}} d z+\int_{L}^{L+L^{\prime}} u_{b}^{(\text {beyond } a)} d z\right] \text {. }
\end{aligned}
$$

\section{B. Membrane strains and membrane energy}

The membrane strain in the circumferential direction is related to the ratio of radii by $\varepsilon_{\theta}=r /\left[R_{\text {finger }}+(t / 2)\right]-1$ at any point $[r(z), z]$ on the surface. For different parts, it is given by

(1) Part $c d$ (and beyond): $\varepsilon_{\theta}=0$.

(2) Part $b c: \varepsilon_{\theta}=\left(R \pm \sqrt{R^{2}-z^{2}}\right) /\left[R_{\text {finger }}+(t / 2)\right]$, where \pm are for the upper and lower halves, respectively.

(3) Part $a b$ : $\quad \varepsilon_{\theta}=[R+(t / 2)+(R-t / 2) \cos (\pi z / L)] /$ $\left[R_{\text {finger }}+(t / 2)\right]$.

(4) The "straight" part beyond $a: \varepsilon_{\theta}=t /\left[R_{\text {finger }}+(t / 2)\right]$. 


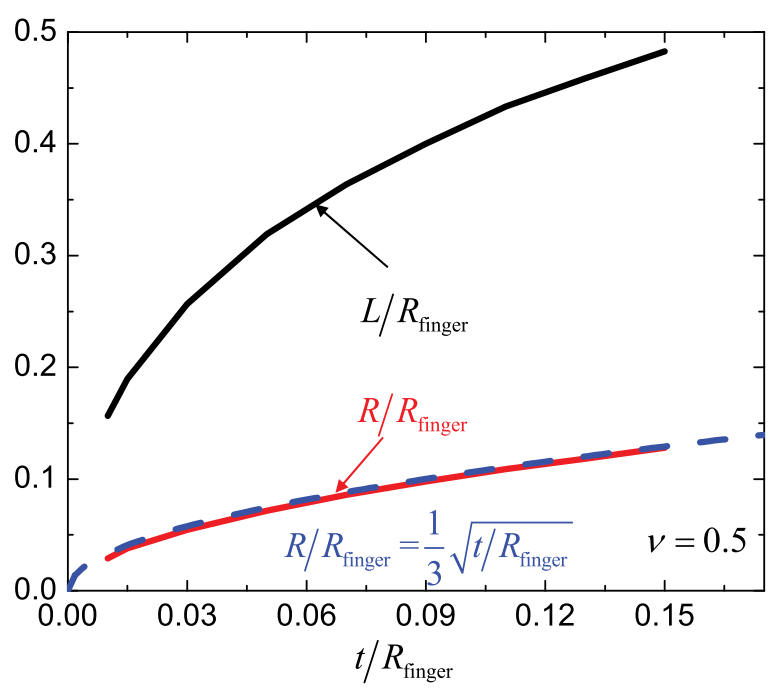

FIG. 4. Normalized length $L$ and radius $R$ versus normalized ecoflex thickness.

As shown in the Appendix, the membrane strain along the meridional direction is $\varepsilon_{s}=-\nu \varepsilon_{\theta}$.

The membrane energy density is ${ }^{17}$

$$
u_{m}=\frac{E t}{2\left(1-\nu^{2}\right)}\left(\varepsilon_{s}^{2}+\varepsilon_{\theta}^{2}+2 \nu \varepsilon_{s} \varepsilon_{\theta}\right)
$$

The membrane energy in the shell is the integration of $u_{m}$ over the area of finger-tube, and is given by

$$
\begin{aligned}
& U_{m}=2 \pi\left(R_{\text {finger }}+\frac{t}{2}\right) *\left[\int_{0}^{L+L^{\prime}} u_{m}^{(c d)} d z\right.
\end{aligned}
$$

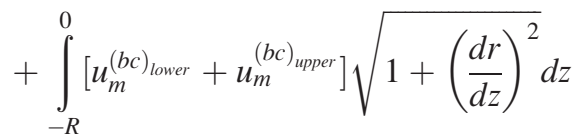

$$
\begin{aligned}
& \left.+\int_{0}^{L} u_{m}^{(a b)} \sqrt{1+\left(\frac{d r}{d z}\right)^{2}} d z+\int_{L}^{L+L^{\prime}} u_{m}^{(\text {beyond } a)} d z\right] \text {. }
\end{aligned}
$$

\section{Energy minimization}

The total energy $U_{\text {total }}$, which consists of bending and membrane energy, depends on $L$ and $R$ since $L^{\prime}$ is obtained (in terms of $L$ and $R$ ) from Eq. (1) for a fixed total length. They are determined by minimizing the total energy, i.e.,

$$
\frac{\partial U_{\text {total }}}{\partial R}=\frac{\partial U_{\text {total }}}{\partial L}=0
$$

which are solved numerically. For an incompressible fingertube ( $\nu=0.5), R$ and $L$, normalized by $R_{\text {finger }}$, depend only on the normalized thickness $t / R_{\text {finger }}$, and is independent of the total length $L_{\text {total }}$ for $L_{\text {total }} \gg L, R$. They are shown in Fig. 4, and the radius $R$ is well approximated by

$$
R=\frac{1}{3} \sqrt{R_{\text {finger }} t}
$$

Figure 5 compares the profile of finger-tube obtained analytically from energy minimization to that obtained by FEM (see the Appendix for details of FEM) for $R_{\text {finger }}$ $=7.5 \mathrm{~mm}$ and $t=0.5 \mathrm{~mm}$ as in experiments. ${ }^{15}$ The good agreement in Fig. 5 provides validation of the analytic model.

\section{Maximum strain in the finger-tube}

Figure 6 gives the strain distribution in the meridional direction on the inner and outer surfaces of the flipped finger-tube obtained by FEM for $R_{\text {finger }}=7.5 \mathrm{~mm}$ and $t=0.5 \mathrm{~mm}$. Its maximum tensile and compressive strains are $35.1 \%$ and $-46.9 \%$, respectively. They agree well with the maximum tensile strain $\varepsilon_{\max }$ and compressive strain $\varepsilon_{\min }$ obtained from the analytic model

$$
\varepsilon_{\max }=\frac{3}{2} \sqrt{\frac{t}{R_{\text {finger }}}} \text { and } \varepsilon_{\min }=-\frac{3}{2} \sqrt{\frac{t}{R_{\text {finger }}}}-\frac{2 \sqrt{R_{\text {finger }} t}}{3\left(2 R_{\text {finger }}+t\right)},
$$

which give the maximum tensile and compressive strains $38.7 \%$ and $-47.1 \%$, respectively.

\section{MAXIMUM STRAIN IN THE ELECTROTACTILE STIMULATOR}

The maximum strains in the electrotactile stimulator during the "flipping-over" process are obtained in this section. The bending moments $M$ and membrane forces $F$ are related to the curvatures and membrane strains given in Sec. II as ${ }^{17}$

$$
\begin{aligned}
& \left\{\begin{array}{l}
M_{s} \\
M_{\theta}
\end{array}\right\}=\frac{E t^{3}}{18}\left\{\begin{array}{l}
2\left(\kappa_{s}-\kappa_{s 0}\right)+\kappa_{\theta}-\kappa_{\theta 0} \\
\kappa_{s}-\kappa_{s 0}+2\left(\kappa_{\theta}-\kappa_{\theta 0}\right)
\end{array}\right\} \text { and } \\
& \left\{\begin{array}{l}
F_{s} \\
F_{\theta}
\end{array}\right\}=\frac{2 E t}{3}\left\{\begin{array}{l}
2 \varepsilon_{s}+\varepsilon_{\theta} \\
\varepsilon_{s}+2 \varepsilon_{\theta}
\end{array}\right\},
\end{aligned}
$$

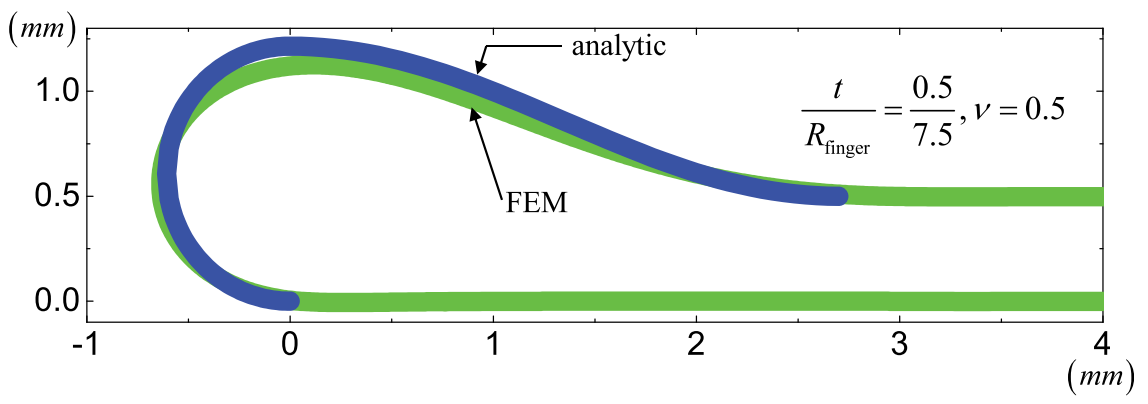

FIG. 5. The profiles of finger-tube given by the analytic model and obtained by FEM. 


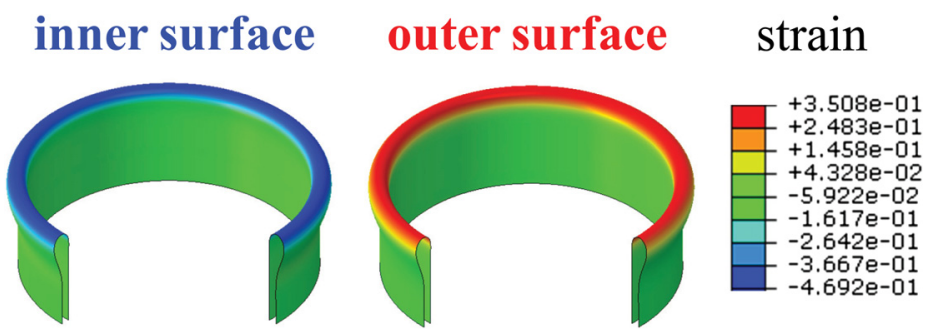

FIG. 6. The strain distribution in the meridional direction on the inner and outer surfaces of the flipped fingertube obtained by FEM.

where the Poisson's ratio for the incompressible finger-tube is $\nu=0.5$. The maximum bending moment and membrane forces are reached in section $b g$ (Fig. 3), and are given by

$$
\begin{aligned}
M_{s, \text { max }} & =2 M_{\theta, \max } \approx-\frac{E t^{2}}{3} \sqrt{\frac{t}{R_{\text {finger }}}}, \quad F_{s, \text { max }} \approx 0 \text { and } \\
F_{\theta, \text { max }} & \approx \frac{2}{3} E t \sqrt{\frac{t}{R_{\text {finger }}}}
\end{aligned}
$$

for $t \ll R_{\text {finger }}$ as in the experiment. ${ }^{15}$

Figure 7 shows two representative cross sections of the electrotactile stimulator on the finger-tube, ${ }^{15}$ which consist of multiple layers of $\mathrm{Au}, \mathrm{Si}$, and PI. Let $n$ denote the total number of layers, with $i=1$ being the finger-tube at the bottom. The Young's modulus, Poisson's ratio and thickness of the $i$ th layers are $E_{i}, \nu_{i}$, and $t_{i}$, respectively, and $E_{1}=E$, $\nu_{1}=0.5$ and $t_{1}=t$. The coordinate $y$ has its origin at the bottom (Fig. 7). The strains in the electrotactile stimulator can be generally written as

$$
\bar{\varepsilon}_{s}=\bar{\kappa}_{s} y+\bar{\varepsilon}_{s 0} \quad \text { and } \quad \bar{\varepsilon}_{\theta}=\bar{\kappa}_{\theta} y+\bar{\varepsilon}_{\theta 0},
$$

where $\bar{\kappa}_{s}$ and $\bar{\kappa}_{\theta}$ are curvatures of the electrotactile stimulator, and $\bar{\varepsilon}_{s 0}$ and $\bar{\varepsilon}_{\theta 0}$ are the strains at the bottom $(y=0)$. The stresses in the $i$ th layer are given by $\bar{\sigma}_{s}=\left(\bar{\varepsilon}_{s}+\nu_{i} \bar{\varepsilon}_{\theta}\right)$ $E_{i} /\left(1-\nu_{i}^{2}\right)$ and $\bar{\sigma}_{\theta}=\left(\nu_{i} \bar{\varepsilon}_{s}+\bar{\varepsilon}_{\theta}\right) E_{i} /\left(1-\nu_{i}^{2}\right)$, which give the membrane forces on the electrotactile stimulator as

$$
\begin{aligned}
\left\{\begin{array}{l}
F_{s, \max } \\
F_{\theta, \max }
\end{array}\right\}= & \int_{0}^{\sum_{i=1}^{n} t_{i}}\left\{\begin{array}{l}
\bar{\sigma}_{s} \\
\bar{\sigma}_{\theta}
\end{array}\right\} d y=\left\{\begin{array}{ll}
K_{11} & K_{12} \\
K_{12} & K_{11}
\end{array}\right\}\left\{\begin{array}{l}
\bar{\varepsilon}_{s 0} \\
\bar{\varepsilon}_{\theta 0}
\end{array}\right\} \\
& +\left\{\begin{array}{ll}
K_{13} & K_{14} \\
K_{14} & K_{13}
\end{array}\right\}\left\{\begin{array}{l}
\bar{\kappa}_{s} \\
\bar{\kappa}_{\theta}
\end{array}\right\},
\end{aligned}
$$

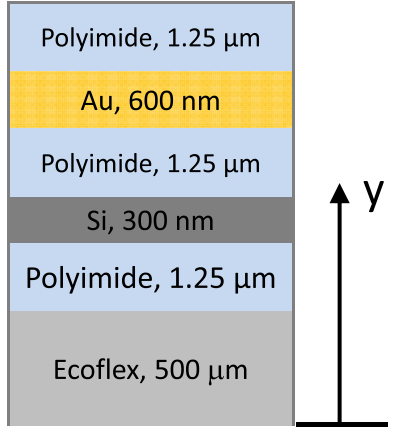

(a)

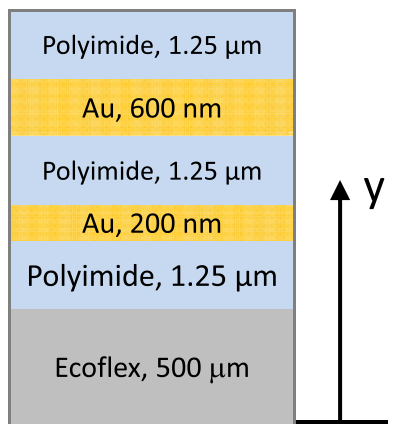

(b)
FIG. 7. Two representative cross sections of the electrotactile stimulator on the finger-tube. where the maximum membrane forces in section $b g$ given in Eq. (10) are used, $K_{11}=\sum_{i=1}^{n} E_{i} t_{i} /\left(1-\nu_{i}^{2}\right), K_{12}$ $=\sum_{i=1}^{n} \nu_{i} E_{i} t_{i} /\left(1-\nu_{i}^{2}\right), K_{13}=\sum_{i=1}^{n} E_{i} t_{i}\left[\left(\sum_{j=1}^{i} t_{j}\right)-\left(t_{i} / 2\right)\right] /$ $\left(1-\nu_{i}^{2}\right)$ and $K_{14}=\sum_{i=1}^{n} \nu_{i} E_{i} t_{i}\left[\left(\sum_{j=1}^{i} t_{j}\right)-\left(t_{i} / 2\right)\right] /\left(1-\nu_{i}^{2}\right)$. Similarly, the bending moments around the bottom $(y=0)$ in section $b g$ can be obtained from the stresses by

$$
\begin{aligned}
\left\{\begin{array}{l}
M_{s, \text { max }}+\frac{1}{2} F_{s, \max } t \\
M_{\theta, \max }+\frac{1}{2} F_{\theta, \max } t
\end{array}\right\}= & \int_{0}^{\sum_{i=1}^{n} t_{i}}\left\{\begin{array}{l}
\bar{\sigma}_{s} \\
\bar{\sigma}_{\theta}
\end{array}\right\} y d y \\
= & \left\{\begin{array}{ll}
K_{13} & K_{14} \\
K_{14} & K_{13}
\end{array}\right\}\left\{\begin{array}{l}
\bar{\varepsilon}_{s 0} \\
\bar{\varepsilon}_{\theta 0}
\end{array}\right\} \\
& +\left\{\begin{array}{ll}
K_{33} & K_{34} \\
K_{34} & K_{33}
\end{array}\right\}\left\{\begin{array}{l}
\bar{\kappa}_{s} \\
\bar{\kappa}_{\theta}
\end{array}\right\},
\end{aligned}
$$

where $K_{33}=\sum_{i=1}^{n} E_{i} t_{i}\left[\left(\sum_{j=1}^{i} t_{j}\right)^{2}-t_{i} \sum_{j=1}^{i} t_{j}+\left(t_{i}^{2} / 3\right)\right] /\left(1-\nu_{i}^{2}\right)$ and $K_{34}=\sum_{i=1}^{n} \nu_{i} E_{i} t_{i}\left[\left(\sum_{j=1}^{i} t_{j}\right)^{2}-t_{i} \sum_{j=1}^{i} t_{j}+\left(t_{i}^{2} / 3\right)\right] /\left(1-\nu_{i}^{2}\right)$. The solution of $\bar{\varepsilon}_{s 0}, \bar{\varepsilon}_{\theta 0}, \bar{\kappa}_{s}$ and $\bar{\kappa}_{\theta}$ is obtained from Eqs. (12) and (13) as

$$
\left\{\begin{array}{c}
\bar{\varepsilon}_{s 0} \\
\bar{\varepsilon}_{\theta 0} \\
\bar{\kappa}_{s} \\
\bar{\kappa}_{\theta}
\end{array}\right\}=\frac{E t}{6} \sqrt{\frac{t}{R_{\text {finger }}}}\left\{\begin{array}{llll}
K_{11} & K_{12} & K_{13} & K_{14} \\
K_{12} & K_{11} & K_{14} & K_{13} \\
K_{13} & K_{14} & K_{33} & K_{34} \\
K_{14} & K_{13} & K_{34} & K_{33}
\end{array}\right\}^{-1}\left\{\begin{array}{c}
0 \\
4 \\
-2 t \\
t
\end{array}\right\} .
$$

The maximum strains in the electrotactile stimulator can then be obtained from Eq. (11), and they are linearly proportional to $1 / \sqrt{R_{\text {finger }}}$. Figures $8(a)$ and $8(b)$ show the maximum principal strain in $\mathrm{Au}$ and $\mathrm{Si}$ versus $\sqrt{t / R_{\text {finger }}}$ for the two representative cross sections of the electrotactile stimulator in Fig. 7. The Young's moduli and Poisson's ratios are $78 \mathrm{GPa}$ and 0.44 for $\mathrm{Au}, 168 \mathrm{GPa}$ and 0.28 for $\mathrm{Si}, 2.5 \mathrm{GPa}$ and 0.34 for PI, and $60 \mathrm{KPa}$ and 0.5 for Ecoflex, respectively. The numerical results obtained by FEM are also shown, and they agree well with the analytic solution in Eq. (14).

\section{CONCLUDING REMARKS}

Due to their extreme deformability, soft materials, such as the classes of silicones described here, are ideal candidates for the platforms and supporting matrices of devices such as finger-tip electronics (e.g., tactile sensors and electrotactile stimulators). The process fabrication, and sometimes use, involves very large levels of mechanical deformation. The analytic models presented here give maximum strains in the 
(a)

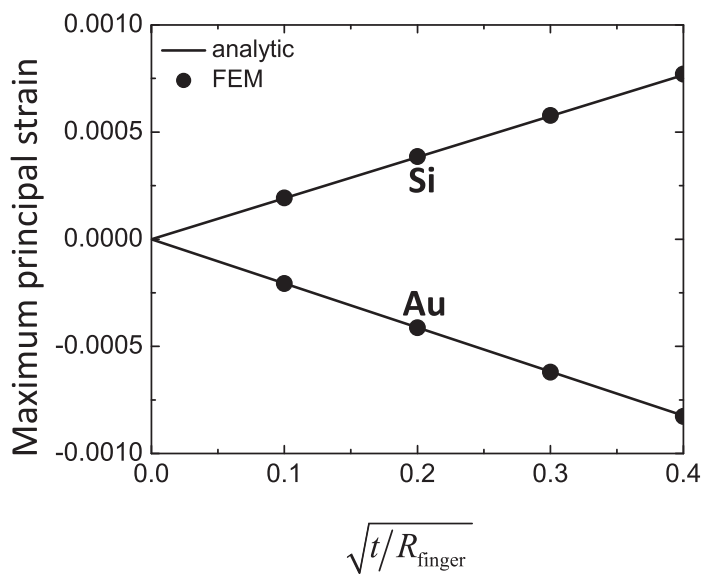

(b)

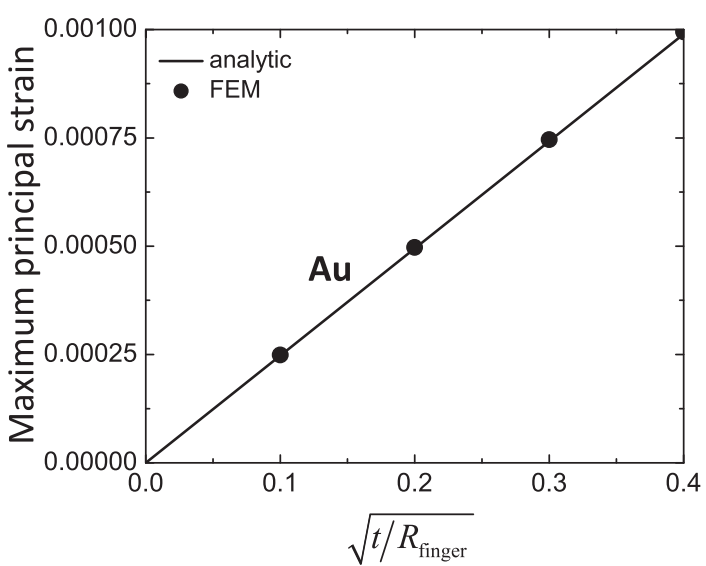

FIG. 8. The maximum principal strains in $\mathrm{Au}$ and $\mathrm{Si}$ in the electrotactile stimulator versus the normalized finger-tube radius.

finger-tube as well as in the electrotactile stimulators, as representative of wide ranging component devices that can be integrated into these systems. The maximum strains are inversely proportional to the square root of finger-tube radius, i.e., $\propto 1 / \sqrt{R_{\text {finger }}}$. The analytic model, validated by FEM, can be used to guide optimal design of fingertip electronics with tactile sensors and electrotactile stimulators and other soft, three-dimensional devices.

\section{ACKNOWLEDGMENTS}

Y.H. and J.A.R. acknowledge the support from NSF (Grant No. INSPIRE 1242240 and the Center for Nanoscale Chemical Electrical Mechanical Manufacturing Systems, which was funded by National Science Foundation under grant CMMI-0328162). K.C.H. acknowledges the support from NSFC.

\section{APPENDIX: THE MEMBRANE STRAIN}

The finite element method is used simulate the "flipping-over" process of the finger-tube. The finger-tube is modeled as an elastic axisymmetric shell by the element SAX1 (2-node linear axisymmetric thin or thick shell) in the finite element program ABAQUS, ${ }^{18}$ interacting with a rigid finger. The finger-tube is flipped over twice, as indicated in Figs. 1(a)-1(c) and Figs. 1(c)-1(e), respectively. In each "flipping-over" process one end of the finger-tube is fixed, whereas the other end is pulled outward and toward the fixed end. The prescribed displacements at the ends are completely relaxed after the first "flipping over" process illustrated in Figs. 1(a)-1(c). The numerical simulations account for contact between the finger-tube and finger, and self-contact between the flipped finger-tube and that on the finger.

Numerical results show that the membrane force $F_{s}$ along the meridional direction is much smaller than the membrane force $F_{\theta}$ along the circumferential direction. Therefore, the approximation $F_{s} \approx 0$, together with the constitutive relation $F_{s}=(2 E t / 3)\left(2 \varepsilon_{s}+\varepsilon_{\theta}\right)$ in Eq. (9), give $\varepsilon_{s}=-\varepsilon_{\theta} / 2$.

${ }^{1}$ D. W. Sparks, P. K. Kuhl, A. E. Edmonds, and G. P. Gray, "Investigating MESA (multipoint electrotactile speech aid)-Transmission of segmental features of speech," J. Acoust. Soc. Am. 63(1), 246-257 (1978).

${ }^{2}$ I. Matteau, R. Kupers, E. Ricciardi, P. Pietrini, and M. Ptito, "Beyond visual, aural, and haptic movement perception: hMT plus is activated by electrotactile motion stimulation of the tongue in sighted and in congenitally blind individuals," Brain Res. Bull. 82(5-6), 264-270 (2010).

${ }^{3}$ Y. P. Danilov, M. E. Tyler, and K. A. Kaczmarek, "Electrotactile vision: Achievements, problems, and perspective," Int. J. Psychophysiol 69(3), $162-163$ (2008).

${ }^{4}$ K. A. Kaczmarek, J. G. Webster, P. Bachyrita, and W. J. Tompkins, "Electrotactile and vibrotactile displays for sensory substitution systems," IEEE Trans. Biomed. Eng. 38(1), 1-16 (1991).

${ }^{5}$ C. A. Lozano, K. A. Kaczmarek, and M. Santello, "Electrotactile stimulation on the tongue: Intensity perception, discrimination, and crossmodality estimation," Somatosens Mot Res. 26(2-3), 50-63 (2009).

${ }^{6} \mathrm{P}$. Bach-y-Rita, M. E. Tyler, and K. A. Kaczmarek, "Seeing with the brain,” Int. J. Hum.-Comput. Interact. 15(2), 285-295 (2003).

${ }^{7}$ L. A. Jones and N. B. Safter, "Tactile displays: Guidance for their design and application," Hum. Factors 50(1), 90-111 (2008).

${ }^{8}$ N. Vuillerme, N. Pinsault, O. Chenu, J. Demongeot, Y. Payan, and Y. Danilov, "Sensory supplementation system based on electrotactile tongue biofeedback of head position for balance control," Neurosci. Lett. 431(3), 206-210 (2008).

${ }^{9}$ F. Vidal-Verdu and M. Hafez, "Graphical tactile displays for visuallyimpaired people,” IEEE Trans. Neural Syst. Rehabil. Eng. 15(1), 119-130 (2007).

${ }^{10}$ D. H. Kim, N. S. Lu, R. Ma, Y. S. Kim, R. H. Kim, S. D. Wang, J. Wu, S. M. Won, H. Tao, A. Islam, K. J. Yu, T. I. Kim, R. Chowdhury, M. Ying, L. Z. Xu, M. Li, H. J. Chung, H. Keum, M. McCormick, P. Liu, Y. W. Zhang, F. G. Omenetto, Y. G. Huang, T. Coleman, and J. A. Rogers, "Epidermal electronics," Science 333(6044), 838-843 (2011).

${ }^{11}$ D. J. Lipomi, M. Vosgueritchian, B. C. Tee, S. L. Hellstrom, J. A. Lee, C. H. Fox, and Z. Bao, "Skin-like pressure and strain sensors based on transparent elastic films of carbon nanotubes," Nat. Nanotechnol. 6, 788-792 (2011)

${ }^{12}$ J. A. Rogers and Y. G. Huang, "A curvy, stretchy future for electronics," Proc. Natl. Acad. Sci U.S.A. 106, 10875 (2009); 106(39), 16889 (2009).

${ }^{13}$ T. Someya, T. Sekitani, S. Iba, Y. Kato, H. Kawaguchi, and T. Sakurai, “A large-area, flexible pressure sensor matrix with organic field-effect transistors for artificial skin applications," Proc. Natl. Acad. Sci. U.S.A. 101(27), 9966-9970 (2004).

${ }^{14}$ D.-H. Kim, N. Lu, R. Ghaffari, Y.-S. Kim, S. P. Lee, L. Xu, J. Wu, R.-H. Kim, J. Song, Z. Liu, J. Viventi, B. de Graff, B. Elolampi, M. Mansour, M. J. Slepian, S. Hwang, J. D. Moss, S.-M. Won, Y. Huang, B. Litt, and J. A. Rogers, "Materials for multifunctional balloon catheters with capabilities in cardiac electrophysiological mapping and ablation therapy," Nature Mater. 10(4), 316-323 (2011).

${ }^{15}$ M. Ying, A. P. Bonifas, N. Lu, Y. Su, R. Li, H. Cheng, A. Ameen, Y. Huang, and J. A. Rogers, "Silicon nanomembranes for fingertip electronics," Nanotechnology 23(34), 344004 (2012).

${ }^{16}$ I. S. Gradshteyn and I. M. Ryzhik, Table of Integrals, Series, and Products (Academic Press, London, 2007).

${ }^{17}$ S. Timoshenko and S. Woinowsky-Krieger, Theory of Plates and Shells (McGraw-Hill, New York, USA, 1959).

${ }^{18}$ Dassault-Systèmes, Abaqus analysis user's manual v.6.10 (Dassault Systèmes Simulia Corp., Rhode Island, 2010). 